\title{
Alternative application approach on black bean: hair coloring product
}

\author{
Chiraporn Inman ${ }^{1}$, Nattaya Lourith ${ }^{1,2^{*}}$ (i) and Mayuree Kanlayavattanakul ${ }^{1,2}$
}

\begin{abstract}
Background: Black bean is one of the important beans as the second most consumed bean globally after soybean. It has been reported as a sustainable source of anthocyanins, important natural colorants used in food and consumer products. However, industrial uses of black bean anthocyanins in pharmaceutics and cosmetics have rarely been explored. Thus, here, a black bean anthocyanin extract was prepared, quality controlled and developed into an efficient hair coloring product.

Results: Black bean was macerated in hydrochloric ethanol to produce a dark-reddish $1.85 \pm 0.04 \%$ dry extract with total anthocyanin content (TAC) of $1.24 \pm 0.48 \mu \mathrm{g} / \mathrm{ml}$ cyanidin-3-glucoside. The extract was readily dissolved in propylene glycol (25\%), giving a reddish to red-brownish solution at $\mathrm{pH}$ of $4.00-7.00$. The addition of antioxidant significantly $(p=0.000)$ enhanced the stability of the extract in an accelerated stability test $(90.34 \pm 5.32$ from $67.83 \pm 3.45 \%$ TAC remaining). Three base hair gels containing different thickeners were developed to yield clear gels with a viscosity of 101,666.67-447,333.33 cps and a pH of 4.80-4.97. The gel base with the best hair application properties was chosen to mix with 8,10 and $12 \%$ black bean extract to produce similar visually assessed red-brown gels $\left(L^{*}=33.25-33.76\right.$, $a^{*}=0.06-0.09, b^{*}=-0.45-0.38$ and $\left.h^{*}=277.54-283.31\right)$ at 73,733.33-99,666.67 cps and a pH of 5.00-5.01. All of the black bean hair coloring gels were stable under the accelerated test. The $10 \%$ hair gel was tested for its hair staining efficacy. The black bean hair coloring gel was found to efficiently color hair, and the color was maintained through four washings.

Conclusions: An alternative application approach of using black bean as a natural colorant is presented here, including its basic requirements for preparation and quality control from an industrial aspect. The efficient semi-permanent hair coloring product containing black bean is an innovative natural product and is considered as one progress towards a sustainable bio-based economy using a readily available industrial crop, black bean.
\end{abstract}

Keywords: Black bean, Hair coloring, Hair dyeing, Anthocyanins

\section{Background}

Black bean (Phaseolus mungo) is one of the important beans as the second most consumed bean globally after soybean. Furthermore, it has been reported to be a sustainable source of anthocyanins, which could be applied as a natural colorant in food, pharmaceutic and cosmetic products. Natural anthocyanins are known to be

\footnotetext{
*Correspondence: nattayal@mfu.ac.th

2 Phytocosmetics and Cosmeceuticals Research Group, Mae Fah Luang University, Chiang Rai 57100, Thailand

Full list of author information is available at the end of the article
}

successfully prepared by maceration in hydrochloric ethanol. Black bean with a total anthocyanin content (TAC) of $213 \pm 2 \mathrm{mg} / 100 \mathrm{~g}$ was characterized to mostly consisted of delphinidin 3-glucoside, petunidin 3-glucoside and malvidin 3-glucoside [1], and the TAC of black bean is higher than that of red cabbage, one of the important known sources of natural anthocyanins [2]. Thus, black bean could be an economical and feasible source of natural colorant that could be used in a variety of product applications.

Anthocyanins are important natural colorant agents found in food and consumer products due to their 
various health benefits $[3,4]$. Although they tend to lack stability, the addition of antioxidant was reported to prolong their shelf life [5]. Industrial approaches to the use of black bean anthocyanins have been extensively explored in food $[2,6,7]$, but only sparsely for pharmaceutic and cosmetic products. Notably, consumers' awareness of and preferences for bio-based or natural products have been increasing recently.

Natural hair care products are of increasing interest among the consumers and researchers [8] as alternatives to synthetic products. Hair dyeing or hair coloring products generate enormous revenues for industries, among which professional salon and home use demands are increasing year by year [9]. Hair coloring products not only include colorants to change the natural hair color shade, but also are applicable for covering grey hair [10]. Hair coloring products are therefore classified into temporary, semi-permanent and permanent products based on the dyeing mechanism of the colorant molecules. The natural colorants dye hair on temporary or semi-permanent basis. The acidic colorant molecules coat the epicuticle, cuticle and the outermost cortex along the hair shaft. On the other hand, the permanent dyes penetrate and diffuse deeper into hair shaft than the temporary and semi-permanent hair dyes. Later, oxidization and polymerization of the colorant molecules in the hair cortex result in coloring on the hair $[11,12]$. Accordingly, oxidation dyes have better hair coloring abilities than natural hair dyes [12], but there are issues that have been reported concerning the safety of synthetic hair dyes. Thus, preference for natural dyes is increasing [9] as well as consumers' expectations of their safety $[10,13]$. Thus, available industrial crops are sources that can be developed into highly efficient, sustainable products using low-cost technologies for selective applications as multipurpose products that are feasible for agricultural and personal care industrial practices.

Thus, a black bean anthocyanin extract was prepared. The color at different $\mathrm{pH}$ conditions applicable for cosmetic products was examined as a basic information requirement for developing cosmetics. Thereafter, hair coloring gels containing black bean colorant extract were developed and evaluated for their dyeing performance. The developed efficient semi-permanent hair coloring product containing black bean is an innovative natural product and considered as an achievement suitable for a sustainable bio-based economy using an available industrial crop, black bean.

\section{Methods}

\section{Materials}

The solvents used for extraction and quality control were all analytical grade. Those of cosmetic grade were applied for hair coloring gel development.

\section{Black bean colorant extract preparation}

Black bean colorant extract was prepared by a procedure modified from a previously described method $[1,7]$ to fit into industrial practice. In brief, black bean (Makro, Thailand) was ground into a fine powder (sieved with 35 mesh) and then macerated in $0.5 \% \mathrm{HCl}$ in $95 \%$ ethanol $(1: 4, \mathrm{w} / \mathrm{v})$ at $4{ }^{\circ} \mathrm{C}$ for $24 \mathrm{~h}$. The colorant solution was filtered (Whatman no. 1) and concentrated to dryness under vacuo. The extractive yield of the black bean colorant extract was calculated. Preparation of the colorant extract was undertaken 3 times.

\section{Quality control of the colorant extract}

Physicochemical properties in terms of color (CIELAB system); $\mathrm{L}^{*}, \mathrm{a}^{*}, \mathrm{~b}^{*}$ and $\mathrm{h}^{*}$, were determined (CM-700d, Konica Minolta, Japan). Solubility of the extract in water, 95\% ethanol and propylene glycol, the common diluents used for cosmetic and personal care products, was examined. Briefly, the solvent $(20 \mu \mathrm{l})$ was stepwise added into the extract $(5 \mathrm{mg})$ until a clear solution was obtained. The solubility was calculated and the $\mathrm{pH}$ (B200, Qis, Germany) of the solution was examined. The coloration of black bean extract at different $\mathrm{pH}$ conditions was monitored $[10,14]$. Its active content in terms of TAC was analyzed by $\mathrm{pH}$ differential method. In short, the colorant extract $(1000 \mu \mathrm{g} / \mathrm{ml})$ in each buffer $(\mathrm{KCl}, 0.2 \mathrm{M}, \mathrm{pH}$ 1.0; and $\mathrm{C}_{2} \mathrm{H}_{3} \mathrm{O}_{2} \mathrm{Na}, 1 \mathrm{M}, \mathrm{pH}$ 4.5) was UV-vis spectroscopically determined at 520 and $700 \mathrm{~nm}$ (Genesys 10S, Thermo-Fisher, USA). TAC was calculated with the extinction coefficient for cyanidin-3-glucoside as follows:

$$
\begin{aligned}
\text { Absorbance }= & \left(A_{520 \mathrm{~nm}}-A_{700 \mathrm{~nm}}\right) \mathrm{pH} 1.0 \\
& -\left(A_{520 \mathrm{~nm}}-A_{700 \mathrm{~nm}}\right) \mathrm{pH} 4.5
\end{aligned}
$$

and expressed as $\mu \mathrm{g} / \mathrm{ml}$ cyanidin-3-glucoside $[15,16]$. The assay was undertaken in triplicate.

Stability of the black bean colorant extract with or without antioxidant, vitamin $\mathrm{E}$ acetate [5], was assessed under an accelerated stability test $\left(4{ }^{\circ} \mathrm{C}\right.$, and $45{ }^{\circ} \mathrm{C}, 24 \mathrm{~h}$ each) for 4 cycles. Color and TAC following the accelerated test were re-examined $[10,14]$.

\section{Hair coloring gel preparation Hair gel base formula development}

Hair gel base formulas containing DI water, ethanol, propylene glycol, carbomer, vitamin E acetate, disodium EDTA, triethanolamine, phenoxyethanol/ethylhexylglycerin and either xanthan gum, hydroxyethyl cellulose (HEC) or hydroxypropyl methylcellulose (HPMC) were formulated. The appearance, $\mathrm{pH}$, viscosity and color of the gel bases were recorded.

The stabilities of the gel bases were tested with an accelerated condition similar to that used to test the black bean 
extract. A stable gel base with a good texture was selected to be developed into black bean hair coloring gel.

\section{The development of a hair coloring gel containing black bean extract}

The selected gel base was mixed with the black bean colorant extract at different concentrations based on the colorant's solubility and the expected color shade. The formulated back bean hair coloring gels were then assessed for their physicochemical properties and stability as above. The most stable developed product was selected for the wash fastness analysis [10].

Tresses of a certain length and weight $(10 \mathrm{~cm} \times 1.5 \mathrm{~g})$ were bleached with $\mathrm{H}_{2} \mathrm{O}_{2}$ (12\% for $30 \mathrm{~min}$ ) and washed with tap water (6 cycles). The bleached tress was color recorded [17] before a slow application (1 min) of the developed black bean hair coloring gel $(1.5 \mathrm{~g})$ and then blow-dried (Philips Voyager Twist 1400, Thailand) at a distance of $5 \mathrm{~cm}$ from the tress with heat of level 2, followed by color recording. The dried and dyed tress was rinsed with 0.1 g Kodomo Baby Shampoo (Lion, Thailand) in tap water $(300 \mathrm{ml})$, blow-dried and color recorded. This wash fastness cycle was repeated until the determined $L^{*}, a^{*}, b^{*}$ and $h^{*}$ were insignificantly different from the bleached tress.

\section{Statistical analysis}

Data are presented as the mean \pm SD. Statistical analysis was performed using SPSS program version 16.0 for Windows. The parameters were compared and analyzed using one-sample $t$ test and ANOVA test with a significance level of $p<0.05$.

\section{Results and discussion}

\section{Preparation and quality control of black bean colorant} extract

Black bean, a common bean purchased from a grocery store, was macerated in hydrochloric alcohol because it has been reported to be a better solvent than water for an efficient anthocyanin extraction [1, 7]. Ethanol was therefore used instead of methanol due to its greater safety and suitability for the food and cosmetic industries $[18,19]$. The black been colorant extract was obtained at an extractive yield of $1.85 \pm 0.04 \%$. A pH differential method, an accurate and substantive method that is an alternative to HPLC [16], was applied for TAC quantification in the unit of cyanidin-3-glucoside. This quality control procedure will therefore be feasible as a routine practice for the industry as it is a widely commercially adopted quantification method [20]. The TAC of the black bean colorant extract in this study was shown to be $1.24 \pm 0.48 \mu \mathrm{g} / \mathrm{ml}$ cyanidin-3-glucoside, that is, higher than the average range previously reported for water extraction [7]. Color recorded in terms of $\mathrm{L}^{*}$ (0-100; black to white), $\mathrm{a}^{*}(-60$ to +60 ; green to red), $\mathrm{b}^{*}\left(-60\right.$ to +60 ; blue to yellow) and hue $\left(\mathrm{h}^{*}\right)$, the important values used to describe the shade of anthocyanins, were recorded. The reddish-pink $\left(L^{*}=42.86 \pm 1.10\right.$, $\mathrm{a}^{*}=8.82 \pm 0.71$ and $\mathrm{b}^{*}=-0.76 \pm 0.12$ ) black bean anthocyanin colorant extract was stronger in color than found by a previous report [7] and more blueish with regard to $h^{*}(355.04 \pm 0.73)$. The strength of the color conformed to its TAC.

The back bean colorant extract was then further examined with regard to its solubility. The solubility of an extract is one of the basic characteristics required for stability assessment and formulation design. The solubility of the black bean colorant extract was examined in water, 95\% ethanol and propylene glycol, which are used as the most common solvents in cosmetics. The extract dissolved well in 95\% ethanol and propylene glycol (25 and $12.5 \%$, respectively), but was undissolved in water $(0 \%)$. These 2 solvents were then used during the design of the hair coloring product. The CIELAB color parameters of the black bean colorant extract in these 2 solvents were also examined. Although the black bean colorants extract dissolved less well in propylene glycol, the color was a deeper red shade $\left(\mathrm{L}^{*}=48.94 \pm 0.51, \mathrm{a}^{*}=10.02 \pm 0.51\right.$, $\mathrm{b}^{*}=1.09 \pm 0.10$ and $\left.\mathrm{h}^{*}=6.24 \pm 0.90\right)$ than that of $95 \% \quad$ ethanol $\quad\left(L^{*}=52.44 \pm 0.13, \quad a^{*}=8.69 \pm 0.04\right.$, $\mathrm{b}^{*}=1.05 \pm 0.01$ and $\left.\mathrm{h}^{*}=6.91 \pm 0.09\right)$ according to $\mathrm{a}^{*}$.

The coloration of the black bean extract at $\mathrm{pH}$ ranges suitable for cosmetic applications was consequently monitored [14]. Black bean extract in 95\% ethanol and propylene glycol $(5000 \mu \mathrm{g} / \mathrm{ml}, \mathrm{pH} 2.7)$ was adjusted to $\mathrm{pH} 4-7$ using $0.5 \% \mathrm{NaOH}$ and their color was monitored (Fig. 1). The examined color shade in this $\mathrm{pH}$ ranges were used for the design of a grey hair covering product design.

The stability of the black bean anthocyanin extract was evaluated using an accelerated stability test. A reduction of $\mathrm{a}^{*}$ is responsible for $\mathrm{TAC}$ as per $\triangle \mathrm{E}$. In addition, storage of the black bean colorant extract in a dried powder form better maintained the extract's quality. Furthermore, the addition of vitamin $\mathrm{E}$ acetate at $0.04 \%$ obviously enhanced this natural colorant extract's stability (Table 1). Thus, vitamin E acetate was therefore included in the hair coloring product development.

\section{Black bean hair coloring gel development}

Hair coloring formulations are classified into temporary, semi-permanent and permanent colorings with regard to rinse-off persistence and dyeing duration. Natural colorants mostly function as temporary hair dyes by cohesion and adhesion of color molecules onto the epicuticle of the hair shaft. This mechanism is therefore known as a color coating effect. Generally, large colorant molecules with 


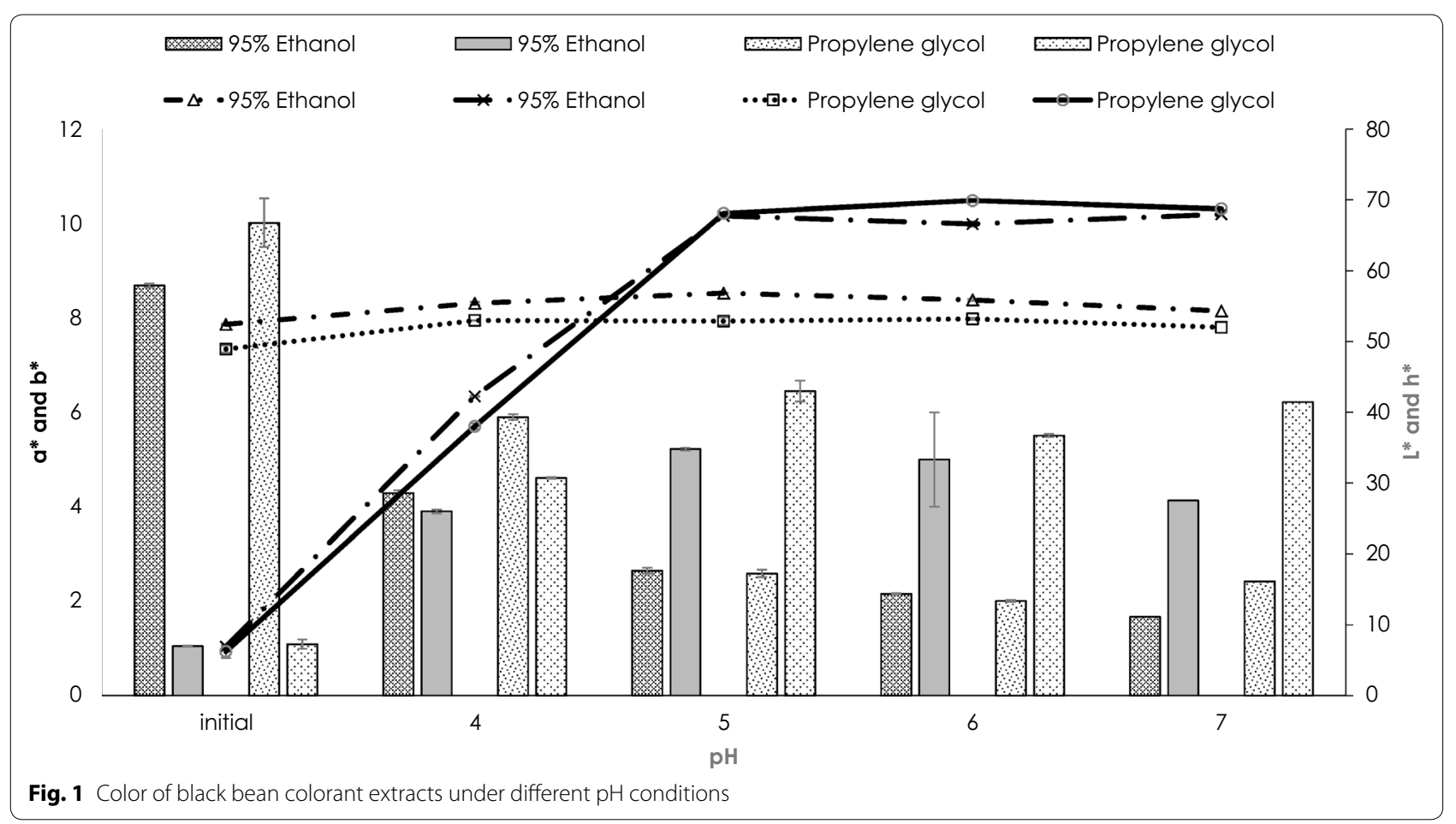

Table 1 Physicochemical stability of black bean anthocyanins extract

\begin{tabular}{|c|c|c|c|c|c|}
\hline & \multirow[t]{3}{*}{ Initial } & \multicolumn{4}{|c|}{ Accelerated stability test } \\
\hline & & \multicolumn{2}{|c|}{ Without antioxidant } & \multicolumn{2}{|c|}{ With antioxidant } \\
\hline & & Powder & Solution & Powder & Solution \\
\hline \multicolumn{6}{|l|}{ Color parameter } \\
\hline$L^{*}$ & $57.02 \pm 0.34$ & $52.53 \pm 0.09$ & $54.48 \pm 0.64$ & $52.46 \pm 0.10$ & $58.80 \pm 0.01$ \\
\hline$a^{*}$ & $3.71 \pm 0.01$ & $1.07 \pm 0.07$ & $0.43 \pm 0.02$ & $1.05 \pm 0.01$ & $0.28 \pm 0.01$ \\
\hline$b^{*}$ & $-0.41 \pm 0.03$ & $3.88 \pm 0.03$ & $1.10 \pm 0.07$ & $3.70 \pm 0.02$ & $1.53 \pm 0.01$ \\
\hline$h^{*}$ & $353.61 \pm 0.38$ & $74.53 \pm 0.03$ & $68.63 \pm 0.68$ & $74.14 \pm 0.22$ & $79.73 \pm 0.49$ \\
\hline$\Delta \mathrm{E}$ & - & $9.02 \pm 0.23$ & $3.74 \pm 0.41$ & $6.70 \pm 0.32$ & $4.33 \pm 0.14$ \\
\hline Visual observed color & Reddish pink & Brown & Pinkish brown & Brown & Pinkish brown \\
\hline Precipitation* & - & + & - & + & - \\
\hline TAC ( $\mu \mathrm{g} / \mathrm{ml}$ cyanidin-3-glucoside) & $1.24 \pm 0.48$ & $0.85 \pm 0.03$ & $0.48 \pm 0.03$ & $1.14 \pm 0.04$ & $0.50 \pm 0.00$ \\
\hline Reduction of TAC (\%) & - & $67.83 \pm 3.45$ & $46.24 \pm 2.86$ & $90.34 \pm 5.32$ & $51.64 \pm 1.15$ \\
\hline
\end{tabular}

Remark*: $(-)$ none, $(+)$ slightly, $(++)$ moderate, $(+++)$ high

an acidic nature dye hair by this mechanism. Semi-permanent hair dyes with smaller acidic molecules permeate or diffuse onto the cuticle and some parts of the cortex of the hair shaft by Vander Waals forces and partially ionic bonds [11, 12]. Thus, hair coloring product design can enhance the color staining efficacy of the natural colorant extract from temporary to a semi-permanent hair dye. Hair coloring products can be prepared in the forms of mousse, stick, gel, cream or spray [12]. Although the natural colorant in temporary dyes for hair is based on its colorant molecule size, the acidic nature of the black bean anthocyanins extract might possibly enable it to function as a semi-permanent dye. Thus, a dosage form of gel that is capable of loading the colorant at a high quantity is of interest and is a challenge to overcome in this study.

Hair coloring gel containing the black bean anthocyanin extract was therefore designed and developed based on its basic characteristics and with the goal of improving the dyeing performance of this natural colorant extract. Hair gel bases containing the ingredients listed in Table 2 
Table 2 Development of base hair coloring gel

\begin{tabular}{|c|c|c|c|}
\hline \multirow[t]{2}{*}{ Ingredients (\% w/w) } & \multicolumn{3}{|l|}{ Formula } \\
\hline & A & B & $\mathrm{C}$ \\
\hline DI water & $\uparrow$ & $\uparrow$ & $\uparrow$ \\
\hline Ethanol & 66.32 & 66.32 & 66.32 \\
\hline Propylene glycol & $\downarrow$ & $\downarrow$ & $\downarrow$ \\
\hline Vitamin E acetate & 0.04 & 0.04 & 0.04 \\
\hline Disodium EDTA & $\uparrow$ & $\uparrow$ & $\uparrow$ \\
\hline Purple spirit & 1.04 & 1.04 & 1.04 \\
\hline Triethanolamine & $\downarrow$ & $\downarrow$ & $\downarrow$ \\
\hline Carbomer & 0.50 & 0.50 & 0.50 \\
\hline Xanthan gum & 0.30 & - & - \\
\hline HEC & - & 0.30 & - \\
\hline HPMC & - & - & 0.30 \\
\hline Phenoxyethanol/ethylhexylglycerin & 1.00 & 1.00 & 1.00 \\
\hline \multicolumn{4}{|l|}{ Initial } \\
\hline $\mathrm{pH}$ & $4.97 \pm 0.02$ & $4.97 \pm 0.01$ & $4.97 \pm 0.02$ \\
\hline Viscosity (cps) & $123,000.00 \pm 13,228.76$ & $304,333.33 \pm 12,423.10$ & $410,666.67 \pm 30,022.21$ \\
\hline$L^{*}$ & $37.24 \pm 0.01$ & $35.76 \pm 0.01$ & $34.71 \pm 0.01$ \\
\hline$a^{*}$ & $-0.10 \pm 0.01$ & $0.06 \pm 0.02$ & $0.04 \pm 0.01$ \\
\hline$b^{*}$ & $0.42 \pm 0.01$ & $-0.47 \pm 0.01$ & $-0.28 \pm 0.01$ \\
\hline$h^{*}$ & $103.36 \pm 1.03$ & $276.98 \pm 2.15$ & $277.45 \pm 0.15$ \\
\hline Visual observed color & Clear light yellow gel & Opaque colorless gel & Opaque colorless gel \\
\hline Precipitation/separation & None & None & None \\
\hline \multicolumn{4}{|l|}{ Accelerated stability test } \\
\hline $\mathrm{pH}$ & $4.87 \pm 0.01$ & $4.84 \pm 0.01$ & $4.80 \pm 0.03$ \\
\hline Viscosity (cps) & $357,333.00 \pm 17,243.00$ & $584,333.00 \pm 16,258.00$ & $575,000.00 \pm 9643.00$ \\
\hline$L^{*}$ & $33.25 \pm 0.02$ & $31.96 \pm 0.02$ & $31.59 \pm 0.28$ \\
\hline$a^{*}$ & $-0.02 \pm 0.03$ & $-0.03 \pm 0.06$ & $-1.08 \pm 0.52$ \\
\hline$b^{*}$ & $0.37 \pm 0.01$ & $-0.51 \pm 0.01$ & $-0.54 \pm 0.42$ \\
\hline$h^{*}$ & $92.78 \pm 3.83$ & $266.41 \pm 6.34$ & $203.61 \pm 13.24$ \\
\hline$\Delta \mathrm{E}$ & $3.99 \pm 0.03$ & $3.81 \pm 0.02$ & $3.36 \pm 0.42$ \\
\hline Visual observed color & Clear light yellow gel & Opaque colorless gel & Opaque colorless gel \\
\hline Precipitation/separation & None & None & None \\
\hline
\end{tabular}

were firstly developed. Water and ethanol function as diluent and solubilizer while propylene glycol works as a humectant in addition to performing its solvent task. Although black bean anthocyanins extracts have been reported to exhibit antioxidant activity [21], the addition of an antioxidant will enhance its stability [5]. In this present study, vitamin $\mathrm{E}$ acetate at $0.04 \%$ was found to enhance the stability of this natural colorant extract as described above. Thus, this antioxidant was selected and included in the gel base development. Furthermore, the $\mathrm{pH}$ of the preparations was adjusted to 5 using triethanolamine to minimize shifting of the black bean color. Three gelling agents were tested in order to enhance the texture of the base preparations.

These bases were examined for their physicochemical properties and tested with regard to their stability. All the formulated bases were stable. The texture of each base was then examined. Base A had the best texture, whereas base B and C containing HEC and HPMC, respectively, were greasier due to their viscosities. Hair gel base A was therefore selected to be developed into the black bean hair coloring gel.

The black bean anthocyanin extract was incorporated into the selected base at 8,10 and $12 \%$ (A8, A10 and A12), selected after taking into account the compatibility of the colorant extract and the preparation ingredients as well as the shade of color as visually assessed. A greater concentration of colorant extract reduced viscosity of the gel due to the acidic nature of the black bean anthocyanin extract. The viscosity of the preparation was governed by carbomer, for which the $\mathrm{pH}$ of the system affects its hydrogel network. The visual observed colors of the 
Table 3 Development of black bean hair coloring gel

\begin{tabular}{llll}
\hline Physicochemical properties & \multicolumn{2}{l}{ Black bean hair coloring gel } & \\
\cline { 2 - 4 } & A8 & A10 & A12 \\
\hline Initial & & & \\
pH & $5.00 \pm 0.01$ & $5.10 \pm 0.01$ & $5.00 \pm 0.01$ \\
Viscosity (cps) & $111,333.33 \pm 0.02$ & $87,366.67 \pm 4845.21$ & $74,800.00 \pm 1200.00$ \\
$L^{*}$ & $33.76 \pm 0.02$ & $33.25 \pm 0.07$ & $33.55 \pm 0.02$ \\
$a^{*}$ & $0.09 \pm 0.02$ & $0.06 \pm 0.00$ & $0.07 \pm 0.01$ \\
$b^{*}$ & $-0.38 \pm 0.02$ & $-0.45 \pm 0.01$ & $-0.38 \pm 0.01$ \\
$h^{*}$ & $283.31 \pm 2.30$ & $277.54 \pm 0.47$ & $279.80 \pm 0.23$ \\
Visual observed color & Brownish red & Brownish red & Brownish red \\
Precipitation/separation & None & None & None \\
Accelerated stability test & & & $4.43 \pm 0.01$ \\
pH & $4.47 \pm 0.01$ & $4.38 \pm 0.00$ & $83,733.33 \pm 4772.14$ \\
Viscosity (cps) & $119,666.67 \pm 5686.24$ & $98,066.67 \pm 8533.07$ & $31.31 \pm 0.05$ \\
$L^{*}$ & $31.21 \pm 0.05$ & $30.36 \pm 0.01$ & $0.12 \pm 0.01$ \\
$a^{*}$ & $0.50 \pm 0.06$ & $0.09 \pm 0.02$ & $-0.44 \pm 0.77$ \\
$b^{*}$ & $-0.38 \pm 0.10$ & $-1.36 \pm 0.08$ & $285.14 \pm 1.61$ \\
$h^{*}$ & $323.48 \pm 4.13$ & $273.63 \pm 1.06$ & $2.25 \pm 0.04$ \\
$\Delta E$ & $2.60 \pm 0.06$ & $2.96 \pm 0.03$ & Brownish red \\
Visual observed color & Brownish red & Brownish red & None \\
Precipitation/separation & None & None &
\end{tabular}

preparations were similar, although A10 was remarkably different in $\mathrm{b}^{*}$, being more bluish and in harmony with $\mathrm{h}^{*}$, referring to the anthocyanin coloration. All the black bean hair coloring gels were then tested for stability and were found to be stable (Table 3). The A10 gel was selected as the candidate for dyeing efficacy evaluation due to its coloring properties and economic feasibility.

The bleached tress with its analyzed color parameters was used as the reference. The black bean hair coloring gel was then applied to the bleached tress. Color in terms of $\mathrm{L}^{*}, \mathrm{a}^{*}$ and $b^{*}$ was used as the international standard method, with grey hair having a higher $L^{*}(13-83)$ and a lower $a^{*}(2-7)$ and $b^{*}(0-13)$ [17]. In addition, $h^{*}$ was recorded based on the nature of the anthocyanin colorant extract. The black bean hair coloring gel was able to visually darken grey hair (Fig. 2) in accordance with $L^{*}$, $a^{*} b^{*}$, and $h^{*}$.

Wash fastness was then tested. Washing cycles were repeated until the monitored color parameters were insignificantly different from the control bleached tress. The black bean hair coloring gel was able to persist through shampooing at least 4 times. Despite the natural anthocyanin property of only temporarily dyeing hair, this developed black bean hair coloring gel could be classified as a semi-permanent coloring gel based on its color staining ability. Adhesion of the coloring gel on the hair enhances the contact time of the black bean anthocyanin

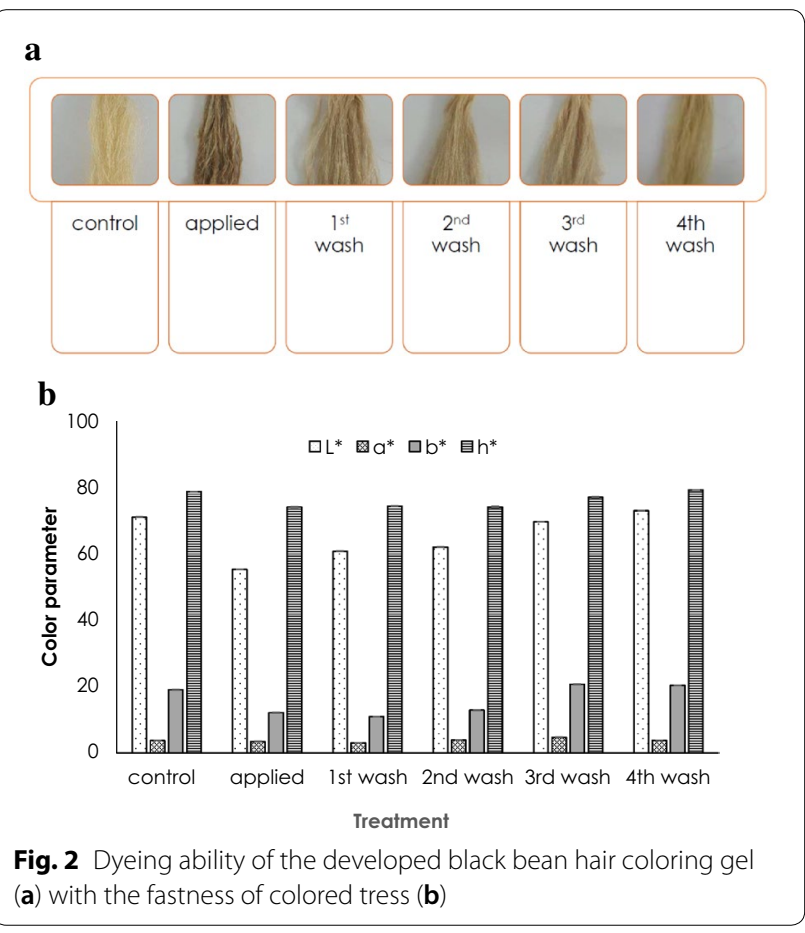

extract, enabling penetration of the colorant molecules into the hair cuticle and partially diffusing throughout the cortex. 


\section{Conclusions}

Black bean can be used as a multipurpose industrial crop for anthocyanin extracts that are applicable for cosmetic preparations. A natural hair coloring product containing black bean colorant extract was shown to be compatible with commonly available ingredients used for topical products. In addition, basic information required for the preparation and quality evaluation of this black bean colorants extract and the developed hair coloring products are presented and are consistent with use in industrial practice. The developed black bean hair coloring gel was shown to be an efficient semi-permanent coloring product for hair. The black bean hair coloring product is an alternative application of this common crop.

\section{Abbreviations}

CIE: Commission Internationale de l'Eclairage; DI water: deionized water; EDTA: ethylenediaminetetraacetic acid; HEC: hydroxyethyl cellulose; HPMC: hydroxypropyl methylcellulose; TAC: total anthocyanins content.

\section{Acknowledgements}

Mae Fah Luang University is acknowledged upon facilities supports during this manuscript preparation. The reviewers are acknowledged for their valuable suggestions that made the article more comprehensive.

\section{Authors' contributions}

CI (M.Sc. student) contributed in all the experimental works, analyzed the data. $\mathrm{NL}$ contributed to project planning, study design and manuscript drafting. MK contributed to co-study design and critical reviewing of the manuscript. All authors read and approved the final manuscript.

\section{Funding}

Not applicable.

\section{Availability of data and material}

Not applicable.

\section{Ethical approval and consent to participate}

Not applicable.

\section{Consent for publication}

Not applicable.

\section{Competing interests}

The authors declared that they have no competing interests.

\section{Author details}

${ }^{1}$ School of Cosmetic Science, Mae Fah Luang University, Chiang Rai 57100, Thailand. ${ }^{2}$ Phytocosmetics and Cosmeceuticals Research Group, Mae Fah Luang University, Chiang Rai 57100, Thailand.

Received: 10 July 2019 Accepted: 17 September 2019 Published online: 21 January 2020

\section{References}

1. Takeoka GR, Dao LT, Full GH, Wong RY, Harden LA, Edwards RH, Berrios HDJ. Characterization of black bean (Phaseolus vulgaris L.) anthocyanins. J Agric Food Chem. 1997:45:3395-400.

2. Prietto L, Mirapalhete TC, Pinto VZ, Hoffmann JF, Vanier NL, Lim L-T, Dias $A R G$, Zavareze EDR. pH-Sensitive films containing anthocyanins extracted from black bean seed coat and red cabbage. LWT Food Sci Technol. 2017:80:492-500.

3. Boo H-O, Hwang S-J, Bae C-S, Park S-H, Heo B-G, Gorinstein S. Extraction and characterization of some natural plant pigments. Ind Crops Prod. 2012;40:129-35.

4. Cortez R, Luna-Vital DA, Margulis D, de Mejia G. Natural pigments: stabilization methods of anthocyanins for food application. Comp Rev Food Sci Food Safety. 2017;16:180-98.

5. Liu S, Liu F, Xue Y, Gao Y. Evaluation on oxidative stability of walnut beverage emulsion. Food Chem. 2016;203:409-16.

6. Aguiler Y, Mojica L, Rebollo-Hernanz M, Berhow M, de Mejía EG, MartínCabrejas MA. Black bean coats: new source of anthocyanins stabilized by $\beta$-clyclodectrin copigmentation in a sport beverage. Food Chem. 2016:212:561-70

7. Mojica L, Berhow M, de Mejia EG. Black bean anthocyanin-rich extras as food colorants: physicochemical stability and antidiabetes potential. Food Chem. 2017;329:628-39.

8. Lourith N, Kanlayavattanakul M. Hair loss and herbs for treatment. J Cosmet Dermatol. 2013;12:210-22.

9. Rose PM, Cantrill V, Benohoud M, Tidder A, Rayner CM, Blackburn RS. Application of anthocyanins from blackcurrant (Ribes nigrum L.) fruit waste as renewable hair dyes. J Agric Food Chem. 2018;66:6790-8.

10. Pipattanamongkol P, Lourith N, Kanlayavattanakul M. The natural approach to hair dyeing product with Cleistocalyx nervosum var. panila. Sus Chem Pharm. 2018;8:88-93.

11. Ballarin B, Galli S, Morigi M. Study on dyeing properties of semipermanent dyestuffs for hair. Int J Cosmet Sci. 2007;29:49-57.

12. Robbins CR. Chemical and physical behavior of human hair. New York: Springer; 2002.

13. Boga C, Delpivi C, Ballarin B, Morigi M, Galli S, Micheletti G, Tozzi S. Investigation on the dyeing power of some organic natural compounds for a green approach to hair dyeing. Dyes Pigm. 2013;97:9-18.

14. Lourith N, Kanlayavattanakul M. Antioxidant color of purple glutinous rice (Oryza sativa) color and its stability for cosmetic application. Adv Sci Lett. 2012;17:302-5.

15. Giusti MM, Wrolstad RE. Characterization and measurement of anthocyanins by UV-Visible spectroscopy. Curr Protocol Food Anal Chem. 2001;F:F1-F1.2.

16. Lee J, Rennaker C, Wrolstad RE. Correlation of two anthocyanin quantification methods: HPLC and spectrophotometric methods. J Food Chem. 2008;110:782-6.

17. Lozano I, Saunier JB, Panhard S, Loussouarn G. The diversity of the human hair colour assessed by visual scales and instrumental measurement. A worldwide survey. Int J Cosmet Sci. 2017;39:101-7.

18. Giusti MM, Jing P. Analysis of anthocyanins. In: Socasiu C, editor. Food colorants: chemical and functional properties. Florida: CRC Press; 2008. p. 479-97.

19. Mercadante AZ, Bobbio FO. Anthocyanins in foods: occurrence and physicochemical properties. In: Socasiu C, editor. Food colorants: chemical and functional properties. Florida: CRC Press; 2008. p. 241-68.

20. Lee J, Durst RW, Wrolstad RE. Determination of total monomeric anthocyanin pigment content of fruit juices, beverage, natural colorants, and wines by $\mathrm{pH}$ differential method: collaborative study. J AOAC Int. 2005;88:1269-78

21. Xu B, Chang SKC. Antioxidant capacity of seed coat, dehulled bean, and whole black soybeans in relation to their distributions of total phenolics, phenolic acids, anthocyanins, and isoflavones. J Agric Food Chem. 2008;56:8365-73.

\section{Publisher's Note}

Springer Nature remains neutral with regard to jurisdictional claims in published maps and institutional affiliations. 